\title{
Solutions of Stiff Systems of Ordinary Differential Equations Using Residual Power Series Method
}

\author{
Mubashir Qayyum (iD) and Qursam Fatima \\ Department of Sciences and Humanities, National University of Computer and Emerging Sciences, Lahore, Pakistan \\ Correspondence should be addressed to Mubashir Qayyum; mubashir.qayyum@nu.edu.pk
}

Received 30 October 2021; Accepted 4 February 2022; Published 4 March 2022

Academic Editor: Clemente Cesarano

Copyright () 2022 Mubashir Qayyum and Qursam Fatima. This is an open access article distributed under the Creative Commons Attribution License, which permits unrestricted use, distribution, and reproduction in any medium, provided the original work is properly cited.

\begin{abstract}
The stiff differential equations occur in almost every field of science. These systems encounter in mathematical biology, chemical reactions and diffusion process, electrical circuits, meteorology, mechanics, and vibrations. Analyzing and predicting such systems with conventional numerical techniques require more time and memory; still accurate solution is completely uneconomical and uncertain. Most of the numerical techniques have stability issues while dealing with stiff systems. To overcome these limitations, residual power series method (RPSM) is proposed for stiff systems of differential equations (DEs). RPSM is applied to various linear and nonlinear stiff systems, and closed-form solutions are achieved. This indicates the effectiveness of proposed scheme for stiff family of DEs. Since this method leads to better results with less computational cost, it can be extended for more complex systems which arise in different areas of engineering and sciences.
\end{abstract}

\section{Introduction}

Stiff system of ordinary differential equations (ODEs) arises in modeling of various real-world physical problems. Solutions of such systems are of great interest for the scientific community. Several numerical, analytical, and seminumerical schemes have been proposed for the solution of such systems. Spijker provided comprehensive review of different stiffness in [1]. He also shows theoretical analysis of numerical methods in stiff situation. Darvishi et al. performed comparative analysis for stiff systems using variation iteration method (VIM) and Adomian decomposition method (ADM) [2]. Abelman and Patidar attempted combustion equation which falls in stiff family of differential equations using finite difference methods [3].Classical numerical techniques, such as finite difference and finite element methods, are computationally expensive and usually affected by round-off errors, which may lead to inaccurate results. Analytical methods are widely used for solving functional equations which are very restrictive for stiff systems and can be used only in special cases $[4,5]$. In 2013, Arqub et al. proposed a method based on Taylor series for the solution of functional equations and systems [6]. This method overcomes the limitation of pure numerical schemes to some extent as it does not require linearization, perturbation, or discretization. Also, it has less computational cost and does not effected by round-off errors. In this method, the solution is considered as the sum of an infinite series, which converges rapidly to the exact solution. This technique is used by many authors for solving linear and nonlinear DEs and systems. Arqub used RPSM to Lane Emden equation (LEE) in [7]. Al-Smadi used this method to different linear and nonlinear IVPs [8]. Mahmood and Yousaf apply the RPSM to Boussinesq-Burgers equations [9]. Kumar and Kumar applied this method to fractional burger type equations in [10]. Kumar et al. also extended this method to fractonal diffusion equation (16). Alquran analyzed fractional form of drainange equation using RPSM [11]. Arqub also altered RPSM to fuzzy DEs [12]. Yao and Kumar applied RPSM to fractional Fokker-Planck equations (21). Kumar and Baleanu apply this scheme to fractional coupled Boussinesq-Burgers equations [13]. El-Ajou et al. used RPSM to nonlinear fractional KdV-Burgers equation [14]. Arqub et al. also apply this method to solve the higher order IVPs [6]. 
Komashynska et al. used this method to solve the system of multi-panto-graph differential equations in [15]. Koashynska et al. also analyzed Fredhlom integral equation with the same method [16].Analysis of stiff systems with traditional numerical techniques requires more time and effort; still accurate solution is uncertain in most of the cases due to stability issues. To overcome these issues, RPSM is proposed for stiff systems in this manuscript. PRSM is tested against various linear and nonlinear stiff systems to check the efficiency and effectiveness of proposed scheme. In the rest of the manuscript, the proposed approach is presented in Section 2, which is then applied to various linear and nonlinear stiff systems in Section 3, while the conclusion is in Section 4.

\section{Residual Power Series Method for Stiff Systems}

In this section, RPSM is explained for the $n^{\text {th }}$-order system of IVPs. Consider the following system:

$$
\begin{array}{r}
\phi_{1}^{\prime}(r)=f_{1}\left(r, \phi_{1}(r), \phi_{2}(r), \ldots, \phi_{n}(r)\right), \\
\phi_{2}^{\prime}(r)=f_{2}\left(r, \phi_{1}(r), \phi_{2}(r), \ldots, \phi_{n}(r)\right), \\
\vdots \\
\phi_{n}^{\prime}(r)=f_{n}\left(r, \phi_{1}(r), \phi_{2}(r), \ldots, \phi_{n}(r)\right),
\end{array}
$$

with initial conditions

$$
\begin{gathered}
\phi_{1}\left(r_{0}\right)=\alpha_{1}, \\
\phi_{2}\left(r_{0}\right)=\alpha_{2}, \\
\cdots \\
\phi_{n}\left(r_{0}\right)=\alpha_{n} .
\end{gathered}
$$

where $r \in\left[r_{0}, r_{0}+a\right]$ and $f_{i}:\left[r_{0}, r_{0}+a\right] \times R^{n} \longrightarrow R$ are nonlinear continuous functions of $r, \phi_{1}, \phi_{2}, \ldots, \phi_{n}$ and $\phi_{i}^{\prime} s$ are unknown functions to be determined. $r_{0}$ and $a$ are real finite constants with $a \geq 0$. It is assumed that $f_{i}$ and $\phi$ are analytical functions on the given interval.

Let us assume solution of the form

$$
\phi_{i}(r)=\sum_{j=0}^{\infty} \phi_{i, j} r^{j}, \quad i=1,2, \ldots, n .
$$

where $\phi_{i, j}$ are terms of approximations and given as $\phi_{i, j}(r)$ $=A_{i, j} r^{j}$. When $j=0, \phi_{i, j}(r)$ satisfy the initial conditions (2), where $\phi_{i, j}(r)$ are the initial guesses of $\phi_{i}(r)$, we have $A_{i, 0}=\phi_{i, 0}\left(r_{0}\right)=\phi_{i}\left(r_{0}\right), i=1,2, \ldots, n$.

If we choose $\phi_{i, 0}\left(r_{0}\right)=\phi_{i}\left(r_{0}\right)$ as initial guesses approximations of $\phi_{i}(r)$, then we can calculate $\phi_{i, j}(r)$ for $j=$ $1,2, \cdots$ and approximate the solutions $\phi_{i}(r)$ of system of IVPs (1) by the $k^{\text {th }}$ truncated series

$$
\phi_{i}^{k}(r)=\sum_{j=0}^{k} A_{i, j}\left(r-r_{0}\right)^{j}, \quad i=0,1,2, \ldots, n .
$$

Rewrite the system in the following form

$$
\phi_{i}^{\prime}(r)-f_{i}\left(r, \phi_{1}(r), \phi_{2}(r), \ldots, \phi_{n}(r)\right)=0, \quad i=1,2, \ldots, n .
$$

Using (4) in (5), the $k^{\text {th }}$ residual function is

$$
\begin{aligned}
\operatorname{Res}_{i}^{k}(r)= & {\left[\sum_{j=1}^{k} j A_{i, j}\left(r-r_{0}\right)^{j-1}\right]-f\left[r, \sum_{j=0}^{k} A_{1, j}\left(r-r_{0}\right)^{j},\right.} \\
& \left.\sum_{j=0}^{k} A_{2, j}\left(r-r_{0}\right)^{j}, \ldots, \sum_{j=0}^{k} A_{n, j}\left(r-r_{0}\right)^{j}\right],
\end{aligned}
$$

and the $\infty^{\text {th }}$ residual function is

$$
\operatorname{Res}_{i}^{\infty}(r)=\lim _{k \longrightarrow \infty} \operatorname{Res}_{i}^{k}(r), \quad i=0,1,2, \ldots, n .
$$

This show that $\operatorname{Res}_{i}^{\infty}(r)$ is infinitely many times differentiable at $r=r_{0}$.

$$
\frac{d^{k-n}}{d r^{k-n}} \operatorname{Res}_{i}^{k}\left(r_{0}\right)=0, \quad k=1,2, \cdots,
$$

which is iterative process of obtaining higher order solution.

\section{Application of RPSM to Stiff Systems}

3.1. Test Problem 1. Consider the following linear stiff system [17]:

$$
\begin{aligned}
\phi_{1}^{\prime}(r)= & -2 \phi_{1}(r)+\phi_{2}(r)+2 \operatorname{Sin}(r), \\
\phi_{2}^{\prime}(r)= & -\left(\epsilon^{-1}+2\right) \phi_{1}(r)+\left(\epsilon^{-1}+1\right) \\
& \left(\phi_{2}(r)-\operatorname{Cos}(r)+\operatorname{Sin}(r)\right),
\end{aligned}
$$

with initial conditions

$$
\begin{aligned}
& \phi_{1}(0)=2, \\
& \phi_{2}(0)=3 .
\end{aligned}
$$

Exact solutions of the problem is

$$
\begin{aligned}
& \phi_{1}(r)=2 e^{-r}+\operatorname{Sin}(r), \\
& \phi_{2}(r)=2 e^{-r}+\operatorname{Cos}(r) .
\end{aligned}
$$

Let we suppose $\epsilon=1$. Also, the approximate solution for the given problem is

$$
\begin{aligned}
& \phi_{1}(r)=\sum_{x=0}^{k} A_{x} r^{x}, \quad k=0,1,2, \ldots n-1, \\
& \phi_{2}(r)=\sum_{x=0}^{k} B_{x} r^{x}, \quad k=0,1,2, \ldots n-1 .
\end{aligned}
$$

Using initial condition with $k=0$ in (13) and (14) gives $\phi_{1}(0)=A_{0}=2, \phi_{2}(0)=B_{0}=3$.

Rewrite (9) as

$$
\begin{array}{r}
\phi_{1}^{\prime}(r)+2 \phi_{1}(r)-\phi_{2}(r)-2 \operatorname{Sin}(r)=0, \\
\phi_{2}^{\prime}(r)+3 \phi_{1}(r)-2\left(\phi_{2}(r)-\operatorname{Cos}(r)+\operatorname{Sin}(r)\right)=0 .
\end{array}
$$

Now, plugging the $k^{\text {th }}$ truncated series ((13)-(14)) in (15) and (16), we obtain 


$$
\operatorname{Res}_{1}^{k}(r)=\sum_{x=1}^{k} x A_{x} r^{x-1}+2 \sum_{x=0}^{k} A_{x} r^{x}-\sum_{x=0}^{k} B_{x} r^{x}-2 \operatorname{Sin}(r)
$$

and

$$
\begin{aligned}
\operatorname{Res}_{2}^{k}(r)= & \sum_{x=1}^{k} x B_{x} r^{x-1}+3 \sum_{x=0}^{k} A_{x} r^{x} \\
& -2\left(\sum_{x=0}^{k} B_{x} r^{x}-\operatorname{Cos}(r)+\operatorname{Sin}(r)\right) .
\end{aligned}
$$

To get $1^{\text {st }}$ approximate solution, use (17) and (18) along with (8) with $k=1$. This leads to $A_{1}=-1, B_{1}=-2$. Proceeding in a similar pattern, RPS solution for the given problem is

$$
\begin{aligned}
\phi_{1}(r)= & 2-r+r^{2}-\frac{r^{3}}{2}+\frac{r^{4}}{12}-\frac{r^{5}}{120}+\frac{r^{6}}{360}-\frac{r^{7}}{1680} \\
& +\frac{r^{8}}{20160}-\frac{r^{9}}{362880}+\frac{r^{10}}{1814400}-\cdots, \\
\phi_{2}(r)= & 3-2 r+\frac{r^{2}}{2}-\frac{r^{3}}{3}+\frac{r^{4}}{8}-\frac{r^{5}}{60}+\frac{r^{6}}{720}-\frac{r^{7}}{2520} \\
& +\frac{r^{8}}{13440}-\frac{r^{9}}{181440}+\frac{r^{10}}{3628800}-\cdots, \\
\phi_{1}(r)= & 2 e^{-r}+\operatorname{Sin}(r), \\
\phi_{2}(r)= & 2 e^{-r}+\operatorname{Cos}(r),
\end{aligned}
$$

which is closed-form solution.

3.2. Test Problem 2. Consider the following nonlinear stiff system [17]:

$$
\begin{aligned}
& \phi_{1}^{\prime}(r)=-\left(\epsilon^{-1}+2\right) \phi_{1}(r)+\epsilon^{-1}\left(\phi_{2}(r)\right)^{2}, \\
& \phi_{2}^{\prime}(r)=\phi_{1}(r)-\phi_{2}(r)\left(1+\left(\phi_{2}(r)\right),\right.
\end{aligned}
$$

with initial conditions

$$
\begin{aligned}
& \phi_{1}(0)=1, \\
& \phi_{2}(0)=1 .
\end{aligned}
$$

Exact solutions of the given system are

$$
\begin{aligned}
& \phi_{1}(r)=e^{-2 r}, \\
& \phi_{2}(r)=e^{-r} .
\end{aligned}
$$

Let us suppose $\epsilon=1$. Also, suppose that approximate solution for the given system is

$$
\begin{aligned}
& \phi_{1}(r)=\sum_{x=0}^{k} A_{x} r^{x}, \quad k=0,1,2, \ldots, \\
& \phi_{2}(r)=\sum_{x=0}^{k} B_{x} r^{x}, \quad k=0,1,2, \ldots
\end{aligned}
$$

The values of $A_{0}$ and $B_{0}$ can be computed from (26) and (27) by taking $k=0$ along with initial conditions. $\phi_{1}(0)=A_{0}=1, \phi_{2}(0)=B_{0}=1$

Rewrite (22) and (23) as follows:

$$
\begin{gathered}
\phi_{1}^{\prime}(r)+3 \phi_{1}(r)-\left(\phi_{2}(r)\right)^{2}=0, \\
\phi_{2}^{\prime}(r)-\phi_{1}(r)+\phi_{2}(r)\left(1+\left(\phi_{2}(r)\right)=0 .\right.
\end{gathered}
$$

Plugging the $k^{\text {th }}$ truncated series (26), (27) in (28) and (29), we get

$$
\begin{aligned}
\operatorname{Res}_{1}^{k}(r)= & \sum_{x=1}^{k} x A_{x} r^{x-1}+3 \sum_{x=0}^{k} A_{x} r^{x}-\left(\sum_{x=0}^{k} B_{x} r^{x}\right)^{2}, \\
\operatorname{Res}_{2}^{k}(r)= & \sum_{x=1}^{k} x B_{x} r^{x-1}-\sum_{x=0}^{k} A_{x} r^{x} \\
& +\sum_{x=0}^{k} B_{x} r^{x}\left(1+\sum_{x=0}^{k} B_{x} r^{x}\right) .
\end{aligned}
$$

To obtain $1^{\text {st }}$ approximate solution put $k=1$ in (30) and (31), then use these residual functions in (8) will give $A_{1}=$ -2 and $B_{1}=-1$. Proceeding in this way, the higher order coefficients can be obtained, and the RPS solution is

$$
\begin{aligned}
\phi_{1}(r)= & 1-2 r+2 r^{2}-\frac{4 r^{3}}{3}+\frac{2 r^{4}}{3}-\frac{4 r^{5}}{15}+\frac{4 r^{6}}{45} \\
& -\frac{8 r^{7}}{315}+\frac{2 r^{8}}{315}-\frac{4 r^{9}}{2835}+\frac{4 r^{10}}{14175}-\cdots, \\
\phi_{2}(r)= & 1-r+\frac{r^{2}}{2}-\frac{r^{3}}{6}+\frac{r^{4}}{24}-\frac{r^{5}}{120}+\frac{r^{6}}{720}-\frac{r^{7}}{5040}+\frac{r^{8}}{40320} \\
& -\frac{r^{9}}{362880}+\frac{r^{10}}{3628800}-\cdots, \\
\phi_{1}(r)= & e^{-2 r}, \\
\phi_{2}(r)= & e^{-r} .
\end{aligned}
$$

which is closed-form (exact) solution. 
3.3. Test Problem 3. Consider the following linear stiff system [18]:

$$
\begin{aligned}
& \phi_{1}^{\prime}(r)=-\phi_{1}(r)+95 \phi_{2}(r), \\
& \phi_{2}^{\prime}(r)=-\phi_{1}(r)-97 \phi_{2}(r),
\end{aligned}
$$

with initial conditions

$$
\begin{aligned}
& \phi_{1}(0)=1, \\
& \phi_{2}(0)=1 .
\end{aligned}
$$

Exact solutions of the problem are

$$
\begin{aligned}
& \phi_{1}(r)=\frac{\left(95 e^{-2 r}-48 e^{-96 r}\right)}{47}, \\
& \phi_{2}(r)=\frac{\left(48 e^{-96 r}-e^{-2 r}\right)}{47} .
\end{aligned}
$$

Approximate solution for the given problem is

$$
\begin{aligned}
& \phi_{1}(r)=\sum_{x=0}^{k} A_{x} r^{x}, \quad k=0,1,2, \cdots, \\
& \phi_{2}(r)=\sum_{x=0}^{k} B_{x} r^{x}, \quad k=0,1,2, \cdots .
\end{aligned}
$$

The values of $A_{0}$ and $B_{0}$ can be computed from (39) and (40) by taking $k=0$ along with initial conditions. $\phi_{1}(0)=A_{0}=1, \phi_{2}(0)=B_{0}=1$

Rewriting the given (35) and (36) in the following form:

$$
\begin{aligned}
& \phi_{1}^{\prime}(r)=-\phi_{1}(r)+95 \phi_{2}(r), \\
& \phi_{2}^{\prime}(r)=-\phi_{1}(r)-97 \phi_{2}(r),
\end{aligned}
$$

and plugging the $k^{\text {th }}$ truncated series (39) and (40) in (41) and (42) to get the following residual functions

$$
\operatorname{Res}_{1}^{k}(r)=\sum_{x=1}^{k} x A_{x} r^{x-1}+\sum_{x=0}^{k} A_{x} r^{x}-95 \sum_{x=0}^{k} B_{x} r^{x}
$$

and

$$
\operatorname{Res}_{2}^{k}(r)=\sum_{x=1}^{k} x B_{x} r^{x-1}+\sum_{x=0}^{k} A_{x} r^{x}+97 \sum_{x=0}^{k} B_{x} r^{x} .
$$

To obtain $1^{\text {st }}$ approximate solution put $k=1$ in (43) and (44), then use these residual functions in (8) will give $A_{1}=$ 94 and $B_{1}=-98$. Proceeding in this way, we can obtain higher order coefficients, and RPS solution is

$$
\begin{aligned}
\phi_{1}(r)= & 1+94 r-4702 r^{2}+\frac{451772}{3} r^{3}-\frac{10842718}{3} r^{4}+\frac{1040901308}{15} r^{5}-\frac{49963263164}{45} r^{6}+\frac{4796473264504}{315} r^{7} \\
& -\frac{57557679174238}{315} r^{8}+\frac{5525537200727228}{2835} r^{9} \\
& -\frac{265225785634907324}{14175} r^{10}+\cdots \cdots, \\
\phi_{2}(r)= & 1-98 r+4706 r^{2}-\frac{451780}{3} r^{3}+\frac{10842722}{3} r^{4}-\frac{1040901316}{15} r^{5}+\frac{49963263172}{45} r^{6} \\
& -\frac{137042093272}{9} r^{7}+\frac{57557679174242}{315} r^{8}-\frac{789362457246748}{405} r^{9}+\frac{265225785634907332}{14175} r^{10}+\cdots \cdots \\
\phi_{1}(r)= & \frac{\left(95 e^{-2 r}-48 e^{-96 r}\right)}{47}, \\
\phi_{2}(r)= & \frac{\left(48 e^{-96 r}-e^{-2 r}\right)}{47} .
\end{aligned}
$$

which is closed-form solution.

$$
\phi_{1}^{\prime}(r)=-1002 \phi_{1}(r)+1000\left(\phi_{2}(r)\right)^{2},
$$

$$
\phi_{2}^{\prime}(r)=\phi_{1}(r)-\phi_{2}(r)\left(\left(\phi_{2}(r)\right)^{2}\right.
$$

3.4. Test Problem 4. Consider the following nonlinear stiff system [18]:

with initial conditions 


$$
\begin{aligned}
& \phi_{1}(0)=1, \\
& \phi_{2}(0)=1 .
\end{aligned}
$$

Exact solutions of the given problem is

$$
\begin{aligned}
& \phi_{1}(r)=e^{-2 r}, \\
& \phi_{2}(r)=e^{-r} .
\end{aligned}
$$

Approximate solution for the system is

$$
\begin{aligned}
& \phi_{1}(r)=\sum_{x=0}^{k} A_{x} r^{x}, \quad k=0,1,2, \cdots \\
& \phi_{2}(r)=\sum_{x=0}^{k} B_{x} r^{x}, \quad k=0,1,2, \cdots
\end{aligned}
$$

The values of $A_{0}$ and $B_{0}$ can be computed from (52) and (53) by taking $k=0$ along with initial conditions. $\phi_{1}(0)=A_{0}=1, \phi_{2}(0)=B_{0}=1$

Rewriting the given system in the following form:

$$
\begin{gathered}
\phi_{1}^{\prime}(r)=-1002 \phi_{1}(r)+1000\left(\phi_{2}(r)\right)^{2}=0, \\
\phi_{2}^{\prime}(r)=\phi_{1}(r)-\phi_{2}(r)\left(\left(\phi_{2}(r)\right)^{2}=0,\right.
\end{gathered}
$$

and plugging the $k^{\text {th }}$ truncated series (52) and (53) in (54) and (55) to obtain the following residual functions

$$
\begin{aligned}
\operatorname{Res}_{1}^{k}(r)= & \sum_{x=1}^{k} x A_{x} r^{x-1}+1002 \sum_{x=0}^{k} A_{x} r^{x} \\
& -1000\left(\sum_{x=0}^{k} B_{x} r^{x}\right)^{2}
\end{aligned}
$$

and

$$
\begin{aligned}
\operatorname{Res}_{2}^{k}(r)= & \sum_{x=1}^{k} x B_{x} r^{x-1}-\sum_{x=0}^{k} A_{x} r^{x} \\
& +\sum_{x=0}^{k} B_{x} r^{x}+\left(\sum_{x=0}^{k} B_{x} r^{x}\right)^{2}
\end{aligned}
$$

To obtain $1^{\text {st }}$ approximate solution put $k=1$ in (56) and (57), we use these residual functions in (8) leading to $A_{1}=$ -2 and $B_{1}=-1$. Proceeding in this way, higher order coefficients can easily be found, and the RPS solution is

$$
\begin{aligned}
\phi_{1}(r)= & 1-2 r+2 r^{2}-\frac{4 r^{3}}{3}+\frac{2 r^{4}}{3}-\frac{4 r^{5}}{15}+\frac{4 r^{6}}{45} \\
& -\frac{8 r^{7}}{315}+\frac{2 r^{8}}{315}-\frac{4 r^{9}}{2835}+\frac{4 r^{10}}{14175}-\cdots, \\
\phi_{2}(r)= & 1-r+\frac{r^{2}}{2}-\frac{r^{3}}{6}+\frac{r^{4}}{24}-\frac{r^{5}}{120}+\frac{r^{6}}{720}-\frac{r^{7}}{5040} \\
& +\frac{r^{8}}{40320}-\frac{r^{9}}{362880}+\frac{r^{10}}{3628800}-\cdots,
\end{aligned}
$$

$$
\begin{aligned}
& \phi_{1}(r)=e^{-2 r}, \\
& \phi_{2}(r)=e^{-r} .
\end{aligned}
$$

which is closed-form solution.

3.5. Test Problem 5. Consider the following system [19]:

$$
\begin{aligned}
& \phi_{1}^{\prime}(r)=\phi_{3}(r)-\operatorname{Cos}(r), \\
& \phi_{2}^{\prime}(r)=\phi_{3}(r)-e^{r}, \\
& \phi_{3}^{\prime}(r)=\phi_{1}(r)-\phi_{2}(r),
\end{aligned}
$$

with initial conditions

$$
\begin{aligned}
& \phi_{1}(0)=1, \\
& \phi_{2}(0)=0, \\
& \phi_{3}(0)=2 .
\end{aligned}
$$

Exact solutions of the problem are

$$
\begin{aligned}
& \phi_{1}(r)=e^{r}, \\
& \phi_{2}(r)=\operatorname{Sin}(r), \\
& \phi_{3}(r)=e^{r}+\operatorname{Cos}(r) .
\end{aligned}
$$

Approximate solution for the given problem is

$$
\begin{aligned}
& \phi_{1}(r)=\sum_{x=0}^{k} A_{x} r^{x}, \\
& \phi_{2}(r)=\sum_{x=0}^{k} B_{x} r^{x}, \\
& \phi_{3}(r)=\sum_{x=0}^{k} C_{x} r^{x}, \quad k=0,1,2, \cdots
\end{aligned}
$$

The values of $A_{0}, B_{0}$, and $C_{i}$ can be computed from (66) by taking $k=0$ along with initial conditions. $\phi_{1}(0)=A_{0}=1, \phi_{2}(0)=B_{0}=0, \phi_{3}(0)=C_{0}=2$

Rewriting the given IVP in the following form:

$$
\begin{aligned}
\phi_{1}^{\prime}(r)-\phi_{3}(r)+\operatorname{Cos}(r) & =0, \\
\phi_{2}^{\prime}(r)-\phi_{3}(r)+e^{r} & =0, \\
\phi_{3}^{\prime}(r)-\phi_{1}(r)+\phi_{2}(r) & =0 .
\end{aligned}
$$

Plugging the $k^{\text {th }}$ truncated series (66) in (67), (68), and (69) to obtain the following residual functions

$$
\begin{array}{r}
\operatorname{Res}_{1}^{k}(r)=\sum_{x=1}^{k} x A_{x} r^{x-1}-\sum_{x=0}^{k} C_{x} r^{x}+\operatorname{Cos}(r), \\
\operatorname{Res}_{2}^{k}(r)=\sum_{x=1}^{k} x B_{x} r^{x-1}-\sum_{x=0}^{k} C_{x} r^{x}+e^{r},
\end{array}
$$

and 


$$
\operatorname{Res}_{3}^{k}(r)=\sum_{x=1}^{k} x C_{x} r^{x-1}-\sum_{x=0}^{k} A_{x} r^{x}+\sum_{x=0}^{k} B_{x} r^{x} .
$$

For $1^{\text {st }}$ approximate solution, put $k=1$ in (70)-(72), we use these functions in (8) to get the following coefficients:

$$
\begin{aligned}
& A_{1}=1, \\
& B_{1}=1, \\
& C_{1}=1 .
\end{aligned}
$$

Repeat the recursive process to obtain higher order coefficients. Hence, RPS solution is

$$
\begin{aligned}
\phi_{1}(r)= & 1+r+\frac{r^{2}}{2}+\frac{r^{3}}{6}+\frac{r^{4}}{24}+\frac{r^{5}}{120}+\frac{r^{6}}{720}+\frac{r^{7}}{5040} \\
& +\frac{r^{8}}{40320}+\frac{r^{9}}{362880}+\frac{r^{10}}{3628800}+\cdots, \\
\phi_{2}(r)= & r-\frac{r^{3}}{6}+\frac{r^{5}}{120}-\frac{r^{7}}{5040}+\frac{r^{9}}{362880}-\cdots, \\
\phi_{3}(r)= & 2+r+\frac{r^{3}}{6}+\frac{r^{4}}{12}+\frac{r^{5}}{120}+\frac{r^{7}}{5040} \\
& +\frac{r^{8}}{20160}+\frac{r^{9}}{362880}+\cdots \\
\phi_{1}(r)= & e^{r}, \\
\phi_{2}(r)= & \operatorname{Sin}(r), \\
\phi_{3}(r)= & e^{r}+\operatorname{Cos}(r) .
\end{aligned}
$$

which is closed-form solution.

3.6. Test Problem 6. Consider the following nonlinear system [19]:

$$
\begin{aligned}
& \phi_{1}^{\prime}(r)=2\left(\phi_{2}(r)\right)^{2}, \\
& \phi_{2}^{\prime}(r)=e^{-r} \phi_{1}(r), \\
& \phi_{3}^{\prime}(r)=\phi_{2}(r)+\phi_{3}(r),
\end{aligned}
$$

with initial conditions

$$
\begin{aligned}
& \phi_{1}(0)=1, \\
& \phi_{2}(0)=0, \\
& \phi_{3}(0)=2 .
\end{aligned}
$$

Exact solutions of the given problem is

$$
\begin{aligned}
& \phi_{1}(r)=e^{2 r}, \\
& \phi_{2}(r)=e^{r}, \\
& \phi_{3}(r)=r e^{r} .
\end{aligned}
$$

Approximate solution for the given problem is

$$
\begin{aligned}
& \phi_{1}(r)=\sum_{x=0}^{k} A_{x} r^{x}, \\
& \phi_{2}(r)=\sum_{x=0}^{k} B_{x} r^{x}, \\
& \phi_{3}(r)=\sum_{x=0}^{k} C_{x} r^{x}, \quad k=0,1,2, \cdots
\end{aligned}
$$

The values of $A_{0}, B_{0}$, and $C_{i}$ can be computed from (83) by taking $k=0$ along with initial conditions. $\phi_{1}(0)=A_{0}=1, \phi_{2}(0)=B_{0}=1, \phi_{3}(0)=C_{0}=0$.

Rewriting the given system in the following form:

$$
\begin{aligned}
\phi_{1}^{\prime}(r)-2\left(\phi_{2}(r)\right)^{2} & =0, \\
\phi_{2}^{\prime}(r)-e^{-r} \phi_{1}(r) & =0, \\
\phi_{3}^{\prime}(r)-\phi_{2}(r)-\phi_{3}(r) & =0 .
\end{aligned}
$$

Plugging the $k^{\text {th }}$ truncated series (83) in (84), (85), and (86) to obtain the following residual functions:

$$
\begin{aligned}
& \operatorname{Res}_{1}^{k}(r)=\sum_{x=1}^{k} x A_{x} r^{x-1}-2\left(\sum_{x=0}^{k} B_{x} r^{x}\right)^{2}, \\
& \operatorname{Res}_{2}^{k}(r)=\sum_{x=1}^{k} x B_{x} r^{x-1}-e^{-r} \sum_{x=0}^{k} A_{x} r^{x},
\end{aligned}
$$

and

$$
\operatorname{Res}_{3}^{k}(r)=\sum_{x=1}^{k} x C_{x} r^{x-1}-\sum_{x=0}^{k} B_{x} r^{x}-\sum_{x=0}^{k} C_{x} r^{x} .
$$

For $1^{\text {st }}$ approximate solution, put $k=1$ in (87)-(89), we use these functions in (8) to get the following coefficients:

$$
\begin{aligned}
& A_{1}=2, \\
& B_{1}=1, \\
& C_{1}=1 .
\end{aligned}
$$

Repeat the process to get higher order coefficients of the series solution. Hence, RPS solution is

$$
\begin{aligned}
& \phi_{1}(r)=1+2 r+2 r^{2}+\frac{4 r^{3}}{3}+\frac{2 r^{4}}{3}+\frac{4 r^{5}}{15}+\frac{4 r^{6}}{45}+\cdots, \\
& \phi_{2}(r)=1+r+\frac{r^{2}}{2}+\frac{r^{3}}{6}+\frac{r^{4}}{24}+\frac{r^{5}}{120}+\frac{r^{6}}{720}+\cdots, \\
& \phi_{3}(r)=r+r^{2}+\frac{r^{3}}{2}+\frac{r^{4}}{6}+\frac{r^{5}}{24}+\frac{r^{6}}{120}+\cdots, \\
& \phi_{1}(r)=e^{2 r}, \\
& \phi_{2}(r)=e^{r}, \\
& \phi_{3}(r)=r e^{r} .
\end{aligned}
$$


which is closed-form solution.

\section{Conclusion}

In this manuscript, the residual power series method is applied to various linear and nonlinear stiff systems of ODEs and closed-form solutions are obtained. These solutions shows that RPSM is simple and easy to use technique for stiff systems and can effectively be used for wide variety of problems in science and engineering.

\section{Data Availability}

All the relevant data are available within the manuscript.

\section{Conflicts of Interest}

The authors declare that they have no conflicts of interest.

\section{References}

[1] M. N. Spijker, "Stiffness in numerical initial-value problems," Journal of Computational and Applied Mathematics, vol. 72, no. 2, pp. 393-406, 1996.

[2] M. T. Darvishi, F. Khani, and A. A. Soliman, "The numerical simulation for stiff systems of ordinary differential equations," Computers \& Mathematics with Applications, vol. 54, no. 7-8, pp. 1055-1063, 2007.

[3] S. Abelman and K. C. Patidar, "Comparison of some recent numerical methods for initial-value problems for stiff ordinary differential equations," Computers \& Mathematics with Applications, vol. 55, no. 4, pp. 733-744, 2008.

[4] A.-A. Hyder, A. H. Soliman, C. Cesarano, and M. A. Barakat, "Solving schrödinger-hirota equation in a stochastic environment and utilizing generalized derivatives of the conformable type," Mathematics, vol. 9, no. 21, p. 2760, oct 2021.

[5] H. S. Ahmed and Abd-Allah Hyder, "Closed-form solutions of stochastic KdV equation with generalized conformable derivatives," Physica Scripta, vol. 95, no. 6, Article ID 065219, 2020.

[6] O. Abu Arqub, Z. Abo-Hammour, R. Al-Badarneh, and S. Momani, "A reliable analytical method for solving higherorder initial value problems," Discrete Dynamics in Nature and Society, vol. 2013, pp. 1-12, 2013.

[7] O. Abu Arqub, A. El-Ajou, A. S. Bataineh, and I. Hashim, “A representation of the exact solution of generalized lane-emden equations using a new analytical method," Abstract and Applied Analysis, vol. 2013, 2013.

[8] M. H. Al-Smadi, "Solving initial value problems by residua lpower series method," Theoretical Mathematics and Applications, vol. 3, no. 1, pp. 199-210, 2013.

[9] B. A. Mahmood and M. A. Yousif, "A residual power series technique for solving boussinesq-burgers equations," Cogent Mathematics, vol. 4, no. 1, Article ID 1279398, 2017.

[10] K. Amit and S. Kumar, "Residual power series method for fractional burger types equations," Nonlinear Engineering, vol. 5, no. 4, 2016.

[11] M. Alquran, "Analytical solutions of fractional foam drainage equation by residual power series method," Mathematical Sciences, vol. 8, no. 4, pp. 153-160, 2014.

[12] A. Abu Arqub, "Series solution of fuzzy differential equations under strongly generalized differentiability," Journal of
Advanced Research in Applied Mathematics, vol. 5, no. 1, pp. 31-52, 2013.

[13] S. Kumar, A. Kumar, and D. Baleanu, "Two analytical methods for time-fractional nonlinear coupled BoussinesqBurger's equations arise in propagation of shallow water waves," Nonlinear Dynamics, vol. 85, no. 2, pp. 699-715, 2016.

[14] A. El-Ajou, O. A. Arqub, and S. Momani, "Approximate analytical solution of the nonlinear fractional KdV-burgers equation: a new iterative algorithm," Journal of Computational Physics, vol. 293, pp. 81-95, 2015.

[15] M. Al-Smadi, "Analytical approximate solutions of systems of multi-pantograph delay differential equations using residual power-series method," Australian Journal of Basic and Applied Sciences, vol. 12, 2014.

[16] I. Komashynska, M. Al-Smadi, A. Ateiwi, and S. Al-Obaidy, "Approximate analytical solution by residual power series method for system of fredholm integral equations," Applied Mathematics \& Information Sciences, vol. 10, no. 3, pp. 975985, 2016.

[17] H. Aminikhah and M. Hemmatnezhad, "An effective modification of the homotopy perturbation method for stiff systems of ordinary differential equations," Applied Mathematics Letters, vol. 24, no. 9, pp. 1502-1508, 2011.

[18] A. Freihet, S. Hasan, M. Al-Smadi, M. Gaith, and S. Momani, "Construction of fractional power series solutions to fractional stiff system using residual functions algorithm," $A d$ vances in Difference Equations, vol. 2019, no. 1, 2019.

[19] D. D. Ganji, H. Mirgolbabaei, Me. Miansari, and Mo. Miansari, "Application of homotopy perturbation method to solve linear and non-linear systems of ordinary differential equations and differential equation of order three," Journal of Applied Sciences, vol. 8, no. 7, pp. 1256-1261, 2008. 